\title{
EDITORIAL
}

\section{Catching breath: monitoring airway inflammation using exhaled breath condensate}

\author{
O. Holz
}

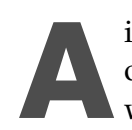
irway inflammation plays a key role in asthma, chronic obstructive pulmonary disease (COPD) and other airway diseases. Noninvasive methods as tools to study these inflammatory processes and to monitor airway diseases are in high demand. This has lead to the rediscovery of sputum analysis and sputum induction as noninvasive tools to obtain samples from the airways [1]. Around this time increased concentrations of nitric oxide (NO) were detected in the exhaled air of asthmatics [2], stimulating interest in breath analysis as an even easier way to assess airway inflammation. The analysis of exhaled breath condensate (EBC) was already known at this time, but only 15 publications related to EBC (between 1990 and 1997) show that only a few researchers used this method as a tool to collect material from the respiratory system. Interest grew steadily, resulting in $\sim 50$ publications in the following 4 yrs and the creation of a joint European Respiratory Society (ERS)/American Thoracic Society (ATS) Task Force at the 2001 ERS meeting in Berlin. This was an early effort to standardise the methodology of collecting and analysing EBC. In this issue of the European Respiratory Journal [3], the leading experts in the field present their final report providing methodological recommendations and a summary of unresolved questions to serve as a guideline for future research.

The appeal of EBC lies in its ability to noninvasively collect a wide range of nonvolatile molecules from the respiratory tract. However, this large number of potentially interesting molecules, which all need to be investigated and validated, seem to be the reason for the rather slow overall progress and the considerable number of open questions listed in the Task Force report [3]. It would probably be helpful now, to rank the open issues according to their overall impact on the methodology. Some issues raised in the report are very important, such as the problem of dilution (which probably affects most biomarkers in EBC), while others, e.g. the influence of ambient air pollutants, seems to be currently a minor issue. Naturally EBC mostly contains water vapour ( $>99.99 \%)$. The fraction of respiratory droplets, which are thought to contain the analytes of interest, were shown to be variable between subjects and within repeated samples of the same subject [4]. Therefore, biomarkers in EBC are often found in concentrations close to the detection limit of available assays. Although frequently argued and stated in the report, it seems unlikely that more sensitive analysers and assays will be able to solve the problem

CORRESPONDENCE: 0. Holz, Research Laboratory, Hospital Großhansdorf, Center for Pneumology and Thoracic Surgery, Woehrendamm 80, D-22927 Grosshansdorf, Germany. Fax: 494102692295 E-mail: 0.holz@pulmoresearch.de of dilution. For most interesting biomarkers, immunological methods (ELISA) are commercially available, which have a detection limit $<10 \mathrm{pg} \cdot \mathrm{mL}^{-1}$, with some even $<1 \mathrm{pg} \cdot \mathrm{mL}^{-1}$. Precautions, such as wearing a face mask to avoid contamination during the handling of these assays are already recommended. Therefore, increasing sensitivities into the $\mathrm{fg}$ $\left(10^{-15}\right) \cdot \mathrm{mL}^{-1}$ range is likely to worsen contamination problems and, at the same time, raise the costs of analysis. It also has to be considered that saliva shares a number of biomarkers with EBC, and although large salivary contaminations are unlikely to occur with the available collection devices, as stated in the report, the use of more sensitive assays could worsen the effects of minimal saliva contaminations.

As nearly all parameters in EBC (except $\mathrm{pH}$ and some biomarkers with a certain degree of volatility, e.g. $\mathrm{H}_{2} \mathrm{O}_{2}$ ) are affected by a potentially variable dilution of respiratory droplets from the epithelial lining fluid there is a renewed interest in markers that could be suitable to correct for this dilution [4]. This has also been a problem for bronchoalveolar lavage measurements, but it has never been satisfactorily solved [5]. There are several dilution indicators under discussion for EBC at the moment, and, interestingly, in a recent publication three of these indicators were used and a similar level of dilution between normal and COPD subjects was found [6].

The Task Force report [2] enables the reader to review the acquired knowledge for each EBC biomarker and its level of validation. It seems that there is a special need for more data on intra-subject and day-to-day variation, both essential for the decision as to whether a biomarker can serve as a research tool, or even has the potential for disease monitoring in clinical practise. Here, the rational for using EBC is similar to that of induced sputum and the analysis of exhaled NO (eNO). Therefore, it seems that EBC should try to profit from the available experience obtained during the development of these methodologies. Induced sputum, for example, is still not very likely to be accepted for clinical practice in outpatient settings or by pneumologists outside larger centres, due to the fact that it remains time consuming and, thus, expensive. Even the convincing evidence that monitoring sputum eosinophils and adjusting therapy accordingly can attenuate the number of asthma exacerbations [7], is, unfortunately, unlikely to change this. So, before starting on this road with any EBC biomarker, it should help to consider this and the fact that adequate reimbursement by health insurances for the analysis of a biomarker always remains as a final hurdle. Conversely, the recent development of the eNO methodology suggests that the chances for any EBC biomarker aiming at a wider clinical use 
will increase with the availability of small, reasonable priced analysers, which provide an instant readout. It has also to be kept in mind that part of the success of eNO seems to be based upon the promotion of NO-analyser manufacturers, which extensively work to raise the interest among outpatient clinics and pneumologist. Finally, it seems to be essential for any noninvasive method to show that its applications make a difference for the patient. This was shown for sputum eosinophils [7] and has recently been published for eNO [8].

So, will we eventually see a study like the one of GREEN et al. [7] or SMITH et al. [8] for a marker of EBC, and which one could be the most likely candidate? Although not undisputed [9], the analysis of EBC $\mathrm{pH}$ is one of the most extensively studied markers and, according to the published literature is shown to be affected in acute asthma, responsive to steroid treatment and reproducible $[10,11]$. The technology to measure $\mathrm{pH}$ and to de-aerate samples prior to measurement is simple, and has been around for a while. Therefore, miniature "online" devices or small desktop set-ups that provide a readout shortly after sampling should pose no major problem. The price tag on such an EBC sampling and $\mathrm{pH}$ analysis set-up could be in the range of modern small NO analysers, which would also be an important criterium for its clinical use. As $\mathrm{pH}$ was shown to be lower not only in asthma, but also in for example cystic fibrosis, COPD and acute lung injury [3], its use as a tool that helps to diagnose asthma like eNO [12] is limited, but the available knowledge suggests an eNO-comparable potential for disease monitoring or the optimisation of treatment.

The publication of the EBC Task Force report [3] is an important step forward that provides the foundation for future research in this field. Time to catch some breath, especially for those engaged in the work-intensive final editing and review process. Time too, however, for focusing on the next steps for those continuing in the development of this method. The large number of outstanding issues will require a coordinated research effort, and a frequent update of the recommendations will be essential for the future of EBC. This could be achieved by continuing the Task Force and discussing progress at regular intervals, such as during ERS or ATS meetings, or within regular workshops, for example, those which recently updated the recommendation for eNO for the third time [13]. Web-based forums could also help to coordinate the research by providing information on issues which are currently under investigation, or to help find partners for cooperation.

In conclusion, the future of exhaled breath condensate will depend to a large extent on the continuing work of enthusiastic researchers, but could be promoted by a suitable environment provided by both the European Respiratory Society and the American Thoracic Society.

\section{REFERENCES}

1 Pin I, Gibson PG, Kolendowicz R, et al. Use of induced sputum cell counts to investigate airway inflammation in asthma. Thorax 1992; 47: 25-29.

2 Alving K, Weitzberg E, Lundberg JM. Increased amount of nitric oxide in exhaled air of asthmatics. Eur Respir J 1993; 6: 1368-1370.

3 Horváth I, Hunt J, Barnes PJ, on behalf of the ATS/ ERS Task Force on Exhaled Breath Condensate. Exhaled breath condensate: methodological recommendations and unresolved questions. Eur Respir J 2005; 26: 523-548.

4 Effros RM, Biller J, Dunning M, Shaker R. Exhaled breath condensate: formation and physicochemical properties. In: Montusci P, ed. New Perspectives in Monitoring Lung Inflammation. Analysis of Exhaled Breath Condensate. Boca Raton, London, New York, Washington DC, CRC Press, 2005; pp. 31-52.

5 Haslam PL, Baughman RP. Report of ERS Task Force: guidelines for measurement of acellular components and standardization of BAL. Eur Respir J 1999; 14: 245248.

6 Effros RM, Peterson B, Casaburi R, et al. Epithelial lining fluid concentrations in chronic obstructive lung disease patients and normal subjects. J Appl Physiol 2005 (In press).

7 Green RH, Brightling CE, McKenna S, et al. Asthma exacerbations and sputum eosinophil counts: a randomised controlled trial. Lancet 2002; 360: 1715-1721.

8 Smith AD, Cowan JA, Brassett KP, Herbison GP, Taylor DR. Use of exhaled nitric oxide measurements to guide treatment in chronic asthma. N Engl J Med 2005; 352: 2173.

9 Effros RM, Hoagland KW, Bosbous M, et al. Dilution of respiratory solutes in exhaled condensates. Am J Respir Crit Care Med 2002; 165: 663-669.

10 Hunt JF, Fang K, Malik R, et al. Endogenous airway acidification. Implications for asthma pathophysiology. Am J Respir Crit Care Med 2000; 161: 694-699.

11 Vaughan J, Ngamtrakulpanit L, Pajewski TN, et al. Exhaled breath condensate $\mathrm{pH}$ is a robust and reproducible assay of airway acidity. Eur Respir J 2003; 22: 889-894.

12 Smith AD, Cowan JO, Filsell S, et al. Diagnosing asthma: comparisons between exhaled nitric oxide measurements and conventional tests. Am J Respir Crit Care Med 2004; 169: 473-478.

13 ATS/ERS recommendations for standardized procedures for the online and offline measurement of exhaled lower respiratory nitric oxide and nasal nitric oxide in adults and children, 2005. Am J Respir Crit Care Med 2005; 171: 912-930. 